\title{
ANÁLISE DE POLÍTICAS PÚBLICAS: DA TÉCNICA ÀS IDEIAS
}

\author{
Ana Cláudia Niedhardt Capella*
}

\begin{abstract}
Resumo: Este estudo tem como objetivo apresentar uma visão panorâmica sobre o campo de políticas públicas, partindo das contribuições seminais de Lasswell, na década de 1950, passando pela "guinada analítica" dos anos 1960 a 1980, até chegar na "guinada argumentativa" que teve início no começo dos anos 1990 e influencia o campo até hoje. Neste percurso, procuramos mostrar que os estudos mais recentes em políticas públicas têm reservado às ideias papel central em seus mecanismos explicativos e com isso incrementado seu poder explicativo.
\end{abstract}

Palavras chave: Políticas Públicas. Produção De Políticas Públicas. Análise De Políticas Públicas. Ideias.

\section{Public Policies analysis: from techniques to ideas}

Abstract: This article intends to offer a panoramic view of the public policy field. We start with Lasswell's seminal study on policy sciences in the 50's, then we move to the "analycentric turn" from the 60's to the 70's and the developments brought by the "argumentative turn" in the early 90's. We intend to demonstrate that the recent studies on public policy field have turn ideas in a central feature of the theoretical models and then enhanced their explanatory power.

Keywords: Public Policy. Policymaking. Public Policy Analysis. Ideas-Based Approach.

\section{Análisis de las políticas públicas: la técnica a las ideas}

Resumen: Este estudio tiene como objetivo presentar una visión panorámica del campo de la política pública, sobre la base de las contribuciones seminales de Lasswell, en el 1950, a través del "giro analítico" de los años 1960 a 1980, hasta llegar al "giro argumentativo", que comenzó a principios de 1990 e influencia en el campo hoy en día. De esta manera, tratamos de mostrar que los estudios más recientes

* Professora e pesquisadora do Departamento de Administração Pública da Unesp, campus de Araraquara.

Recebido em: 15/01/2014 - Aceito em: 01/06/2014. 
en las políticas públicas han reservado las ideas papel central en sus mecanismos explicativos y con ello el aumento de su poder explicativo.

Palabras-clave: Políticas Públicas. Producción de las Políticas Públicas. Análisis de Políticas Públicas. Ideas.

\section{Introdução}

O papel das ideias é hoje central para os estudos em políticas públicas. Desde os anos 1990, diversos autores apontaram os limites e contradições da análise tecnocrática e racionalista de políticas públicas, característica do campo desde seu surgimento, nos anos 1950, e mostraram que as políticas são feitas de ideias. Considerando as ideias como crenças, valores, visões de mundo ou entendimentos compartilhados, a literatura especializada aponta que a disputa sobre as ideias está no centro do processo de produção de políticas públicas. Os autores que destacam o papel das ideias enfatizam a centralidade do discurso, da interpretação, da representação simbólica, entendendo que a produção de políticas se aproxima mais do processo de argumentação do que de técnicas formais de solução de problemas.

Este estudo tem como objetivo retomar a trajetória do campo de políticas públicas a partir da inflexão trazida pela análise baseada nas ideias. Para tanto, apresentaremos de forma sintética o histórico do campo, desde o momento inicial da área, as policy sciences, passando pela chamada "guinada analítica" para chegarmos à "guinada argumentativa". Por fim, destacaremos algumas contribuições teóricas mais recentes, apresentando três modelos já estabelecidos e difundidos no campo e que inseriram com sucesso as ideias em seus mecanismos explicativos.

\section{Das policy sciences à análise das políticas públicas: origens do campo}

Estudiosos do campo de estudos e pesquisas em políticas públicas localizam o surgimento da área nos anos 1950, nos 
Estados Unidos, sob o signo das policy sciences (Lasswell, 1951) ${ }^{1}$. Embora o estudo sobre o poder político e seu exercício tenha ocupado espaço central nas discussões de áreas mais tradicionais como a filosofia e a ciência política, o movimento liderado por Harold Lasswell, buscava estabelecer métodos científicos especificamente voltados para o estudo sistemático das questões relacionadas às práticas de governo ${ }^{2}$. O projeto das policy sciences visava à construção de um campo de conhecimento projetado especificamente para o desenvolvimento de pesquisas e métodos de enfrentamento dos problemas governamentais, ou seja, um campo orientado para a resolução de problemas concretos relacionados às mais diversas políticas públicas. Tal campo também seria caracterizado por uma perspectiva essencialmente multidisciplinar, apoiandose em disciplinas consideradas úteis para o enfrentamento dos problemas governamentais. Outra característica das policy sciences consistia em um esforço acadêmico voltado ao desenvolvimento de métodos e teorias sofisticados, envolvendo análises rigorosas baseadas em métodos quantitativos capazes de viabilizar a investigação sobre as relações governamentais e projetar soluções para os problemas públicos diagnosticados na realidade empírica.

${ }^{1}$ Para uma análise histórica sobre as origens do campo, consultar DeLeon (2008).

${ }^{2}$ É importante notar que, já no final do século XIX e início do século XX, estrutura-se uma disciplina voltada para as mesmas preocupações: a administração pública. Essa área surgiu, como campo de estudos e como área de formação de servidores públicos, nos EUA. Apesar de a administração pública ser originalmente estruturada como subárea da ciência política, sua trajetória inicial foi marcada por uma preocupação em distanciar-se da política e identificar-se com métodos e técnicas da administração científica, conjunto de práticas de administração industrial que surgiram no mesmo período. Nos anos que se seguiram, a disciplina vivenciou uma relação inconstante com o campo da ciência política, tendo praticamente distanciado-se dela entre os anos 1950 e 1970. É neste período que surge a proposta de Lasswell, que mantém o campo de políticas públicas no interior da ciência política. Para uma competente análise da relação entre administração púbica e ciência política, consultar Farah (2013). 
Por fim, o campo das policy sciences pretendia-se orientado para valores democráticos (DeLeon, 2008; Smith e Larimer, 2009). As policy sciences seriam, assim, uma ciência social voltada para a produção de conhecimento direcionado à resolução de problemas governamentais por meio da expertise de técnicos treinados em métodos de análise e solução de problemas. Tais indivíduos poderiam identificar problemas concretos na sociedade, mantendo a neutralidade técnica em suas decisões e ações, facilitando o trabalho de políticos e administradores públicos.

Muitas das premissas das policy sciences mostraram-se falhas por diferentes motivos, entre os quais algumas contradições presentes no próprio projeto. A visão elitista sobre o processo de produção de políticas - a criação e manutenção de uma elite treinada, detentora de conhecimento, técnicas, métodos e ferramentas para a resolução de problemas governamentais - pode ser entendida como potencialmente conflituosa com a ideia de fortalecimento dos valores democráticos, uma vez que restringe a participação dos cidadãos nesse processo. Além disso, como consideram Smith e Larimer (2009), o projeto das policy sciences contém uma tensão permanente entre valores políticos e a almejada objetividade científica. A ciência pressupõe um mundo objetivo, independente do olhar de seu observador, enquanto o mundo da política envolve a percepção do pesquisador, seus valores, crenças ou ideologias.

Science (...) cannot make a policy choice any less political. (...) Lasswell argued that facts would be put into service of democratic values. He never seemed to fully recognize that facts and values could conflict, let alone the values might in some cases determine facts. (Smith e Larimer, 2009: 12).

Apesar das limitações, esse movimento estimulou os primeiros debates sobre a constituição de um campo de conhecimento especificamente voltado ao processo de produção de políticas públicas. Nos anos que se seguiram (anos 1960 em diante), sobretudo no contexto dos Estados Unidos, diversos esforços foram conduzidos no sentido do desenvolvimento de 
técnicas e métodos para a análise de políticas públicas, aplicando conhecimentos oriundos da engenharia, pesquisa em operações, matemática aplicada e economia. Este período foi caracterizado pelo predomínio da técnica, fundamentada na crença de que qualquer problema pode ser resolvido por meio de uma análise minuciosa, compreendendo a decomposição de problemas em suas menores unidades. Essa "guinada analítica" (analycentric turn) (Dunn, 1994) foi alimentada pela rápida expansão e influência de organizações de pesquisa (os think tanks) que fomentaram o emprego de técnicas de análise junto a órgãos governamentais e também junto à comunidade acadêmica. A perspectiva técnica, no entanto, restringe (ou mesmo ignora) os aspectos políticos, sociais e administrativos, presentes na formulação inicial de Lasswell. Nessa abordagem, a política é entendida em termos puramente tecnocráticos: uma política pública é uma solução para um problema governamental, a análise tem como objetivo central a eficiência, com base na identificação do curso de ação mais eficiente possível para um dado problema percebido. A análise de políticas públicas pertenceria ao domínio de experts, tecnocratas, consultores especializados e demais profissionais capazes de oferecer soluções sem se envolverem com o mundo da política.

A partir dos anos 1990, diversos autores apresentaram críticas a essa abordagem, questionando a objetividade do analista de políticas públicas, o cientificismo da área e o caráter estritamente técnico da análise de políticas. Dryzek (1993) sintetiza tais críticas à análise de políticas públicas em dois pontos principais. $O$ primeiro relaciona-se a uma forma específica de entender a ciência, marcada pelo objetivismo presente nas perspectivas desenvolvidas por positivistas. Nessa concepção da ciência, assume-se a existência de um conjunto de regras e procedimentos universalmente válidos para o estabelecimento de relações causais. Segundo o autor, "Positivism in policy analysis can be characterized in terms of a belief that policy interventions should be based on causal laws of society and verified by neutral empirical observation." (Dryzek, 1993, 218). A lógica das políticas públicas, no entanto, não segue tais parâmetros, uma vez que não há leis gerais aplicáveis a fenômenos 
sociais. Além disso, longe de serem claros e indiscutíveis, os objetivos de uma política são sempre ambíguos e mal definidos, passíveis de questionamento, uma vez que os valores que os suportam são distintos, múltiplos e conflituosos. Outro aspecto a considerar é a possibilidade de que ações distintas daquelas originalmente intencionadas na formulação sejam realizadas ao longo da execução de uma política. Ou seja, a distância entre um objetivo definido e aquilo que efetivamente é realizado pode subverter a premissa positivista de causas e efeitos.

O segundo ponto identificado por Dryzek (1993) nas críticas ao modelo da análise de políticas relaciona-se a uma perspectiva particular de compreensão do comportamento humano: a racionalidade instrumental (ou, nos termos empregados por Dunn, a "guinada analítica"). Técnicas difundidas na análise de políticas públicas, como análises de custo-benefício, análises decisórias, modelagem de sistemas, programação linear, orgamentoprograma, entre outras, procuram estabelecer padrões normativos para as decisões em políticas públicas. Tais técnicas, aponta o autor, prescrevem cursos de ação baseados em seus próprios métodos, e não em relações causais observáveis empiricamente, contribuindo apenas para ratificar as conclusões do analista e não para testar hipóteses causais. Além disso, e talvez um dos maiores problemas na aplicação dessas ferramentas à análise de políticas públicas, é o fato de que questões e decisões, fundamentalmente políticas, são obscurecidas pelo viés da técnica. As conclusões das análises dependem das alternativas selecionadas, sobre as quais pesam os valores dos analistas durante o processo de escolha.

Em resposta a essas críticas, vários pesquisadores desenvolveram diferentes alternativas de análise e novas perspectivas para o estudo de políticas públicas. Na próxima seção, apresentaremos algumas contribuições importantes para o campo que, ao defenderem a centralidade das ideias na análise das políticas públicas, ficaram conhecidas como a "guinada argumentativa" (argumentative turn) (Fischer e Forester, 1993). 


\section{O estudo das políticas públicas: a guinada argumentativa}

Estabelecidos os limites da análise de políticas calcada no objetivismo e na racionalidade instrumental (Dryzek, 1993), diversos autores destacaram a importância das ideias como elemento fundamental para a compreensão das políticas públicas. A "guinada argumentativa" no campo de políticas públicas insere-se em um debate mais amplo, presente em diferentes áreas das ciências sociais, a partir de meados da década de 1980. Tal perspectiva enfatiza o papel das ideias e é central nas abordagens pós-positivistas - também chamadas pós-modernas, pós-empiricistas ou pós-estruturalistas, abordagem ideacional, entre outras denominações. Nas próximas seções serão apresentadas e discutidas algumas críticas ao modelo racionalista, bem como novas perspectivas propostas por dois importantes autores identificados com a "guinada argumentativa". Mais do que investigar os argumentos contrários à análise tradicional de políticas públicas, buscaremos apontar como esses autores entendem a influência das ideias - narrativas, argumentos e persuasão - na produção de políticas públicas.

\section{Majone: políticas públicas e linguagem}

Uma das principais críticas ao modelo positivista e racionalista de análise de políticas públicas foi desenvolvida por Majone (1989). Para o autor, tal perspectiva sobre a produção de políticas públicas foi encorajada por duas formas distintas de pensar que acabaram se aproximando nos anos 1960 e que estruturaram os estudos iniciais no campo. A primeira delas é o decisionismo, um modelo fortemente tecnocrático baseado na perspectiva do ator racional desenvolvido, originalmente, na economia, e com grande influência sobre administração. A segunda influência origina-se no campo da ciência política, mais especificamente em um modelo político que entende as políticas públicas como o resultado das disputas entre diferentes interesses e pressões no sistema político. 
O decisionismo apresenta a análise de políticas públicas como uma atividade essencialmente técnica, aproximando-se da crítica à racionalidade instrumental de Dryzek. Nessa perspectiva, poucos atores políticos são encarregados de produzir as políticas por meio de escolhas processadas de forma racional. Tal lógica de decisão influenciou os estudos sobre administração desde o final da década de 1930, sobretudo nas pesquisas relacionadas às operações industriais e militares. Nos anos 1950, a perspectiva decisionista foi incorporada ao campo nascente de políticas públicas, no qual caberia ao analista a aplicação métodos científicos de forma a maximizar os resultados das políticas. Para Majone (1989), naquele momento, as situações investigadas pelos pesquisadores encaixavam-se bem no método de decisão racional, pois envolviam poucos participantes familiarizados com as questões, numa estrutura de comando bem definida. Nesse período, o papel dos analistas e dos tomadores de decisão era razoavelmente claro:

Whether the users of the analysis were high-level military officers or high-level managers, analysis was done primarily or even (because the requirement of military or industrial secrecy) exclusively for them. The analyst did not have to address any audience other than the decision maker, or a small group of decision makes who had commissioned the study. (Majone, 1989: 14).

No entanto, nas décadas seguintes, o contexto da produção de políticas foi substancialmente alterado, seja pela natureza dos problemas ou pelas mudanças nas características organizacionais e políticas nos quais os analistas de políticas operavam. A análise de políticas, antes restrita a um grupo pequeno de especialistas, passa a direcionar-se a uma audiência mais ampla, para a qual as questões técnicas relacionadas à eficiência deixam de ser entendidos como os únicos balizadores das decisões em políticas públicas.

The problems claiming analytic attention were broader and more complex. Strategic, rather than tactical, issued loomed increasingly important, while 
subjective uncertainty was seen to be more crucial then the statistical regularities assumed in earlier models. At the same time, the growing role of analysis in public debates meant that the analysts (...) had to pay attention to questions of equity and feasibility. (Majone, 1989: 14).

A segunda crítica de Majone direciona-se ao modelo político de tomada de decisão, caracterizado pela noção de que o desenvolvimento de uma política é determinado pela configuração dos interesses dominantes. Nessa perspectiva, atores como parlamentares, burocratas e grupos de interesses ligados a diferentes setores de atividade econômica, conduziriam o processo de produção de políticas e as decisões tomadas refletiriam seus interesses. "We miss a great deal", diz o autor, "if we try to understand policy-making solely in terms of power, influence, and bargaining, to the exclusion of debate and argument." (Majone, $1989,02)$. O autor mostra que mesmo quando um ator persegue seus próprios interesses, ele precisa, num sistema político regido pela discussão, justificar suas escolhas numa política, apelando para aspectos técnicos, intelectuais, ou mesmo para a opinião pública. Assim, o modelo político, centrado nos interesses dos atores, não considera o processamento paralelo de debate e argumentação que acompanha todas as fases de uma política. Os atores interessados em uma política procuram coletar evidências empíricas e as organizam de forma a garantir maiores chances de convencimento, na busca por apoio às suas propostas. Indivíduos e grupos envolvidos numa política apoiam-se em análises técnicas para desafiar as propostas de seus oponentes e constroem argumentações que apelam a valores e crenças, bem como aos interesses de outros grupos, de forma a construir suporte para suas propostas. Desta forma, não apenas os interesses dos atores importam para a análise de uma política, mas também o processo de argumentação, persuasão e construção de evidências, uma vez que as "políticas públicas são feitas de linguagem" (Majone, $1989,01)$. 
Se, para o autor, a análise de políticas não se caracteriza pela técnica nem pelos interesses, mas pela habilidade em construir argumentos, qual seria o papel do analista de políticas? Neste aspecto, o analista de políticas se aproximaria de um produtor de argumentos e defensor de ideias:

The policy analyst is a producer of policy arguments, more similar to a lawyer - a specialist in legal arguments - than to an engineer or scientist His basic skills are not algorithmical but argumentative: the ability to probe assumptions critically, to produce and evaluate evidence, to keep many threads in hand, to draw for an argument from many disparate sources, to communicate effectively (Majone, 1989: 21-22).

Assim, para o autor, ao invés de manejar ferramentas técnicas, o analista de políticas públicas atuaria essencialmente na construção de evidências e argumentos para o debate público e persuasão política. Uma evidência, para o autor, não consiste em dado ou informações objetivos, mas sim na seleção desses elementos no processo argumentativo de forma a persuadir o público sobre a veracidade - ou não - de uma alegação. Mais do que solucionar problemas de forma tecnocrática, analisar políticas significa aproximar fato e valor, coletar evidências, desenvolver argumentos e persuadir amplas audiências em torno de ideias.

\section{Stone: Ideias e narrativas}

Outra crítica à concepção original de políticas públicas foi desenvolvida por Stone (2002). Contrária ao que chama de "projeto racionalista", Stone concentra sua análise na questão que considera fundamental à análise do processo político: a dinâmica das ideias.

What I see as the essence of policy making in political communities [is] the struggle over ideas. Ideas are a medium of exchange and a mode of influence even more powerful than money and votes and guns (...). Ideas are at the center of all political conflict. Policy 
making, in turn, is a constant struggle over the criteria for classification, the boundaries of categories, and the definition of ideals that guide the way people behave.

(Stone, 2002: 11).

O "projeto racionalista", para a autora, é composto por três elementos. Em primeiro lugar, o racionalismo constitui-se em um modelo de comportamento - o modelo da escolha racional. E este modelo falha em explicar as decisões complexas tomadas ao longo da produção de uma política, assim como o estudo de Majone (1989) apontou. O segundo elemento do chamado projeto racionalista é um modelo de sociedade baseada no mercado, em que indivíduos efetuam trocas em busca de seu próprio bem estar. A autora procura mostrar que esta perspectiva não contribui para o entendimento das políticas públicas, uma vez que o processo de argumentação em torno de uma política pode ser melhor compreendido em outro modelo de sociedade, baseado na noção de "comunidade política". As comunidades reúnem indivíduos em torno de questões e crenças compartilhadas, ao invés dos indivíduos atomizados presentes na noção de sociedade como mercado. Por fim, o terceiro elemento que integra o "projeto racionalista" é uma visão produtivista sobre as políticas, na qual programas e projetos são desenvolvidos de maneira linear e sequencial. Essa perspectiva está diretamente relacionada à ideia da solução racional de problemas, discutida anteriormente. Sustentado por esses três elementos, o "projeto racionalista" falha em capturar a essência da política que, para a autora, consiste na disputa sobre ideias. E o que são ideias, para a autora? "Each idea is an argument, or more accurately, a collection of arguments in favor of different ways of seeing the world." (Stone, 2002, 11).

Assim como Majone (1989), a autora destaca a importância da argumentação e da linguagem para os estudos em políticas públicas. Ao contrário da perspectiva racionalista, Stone defende que problemas não são simplesmente diagnosticados na realidade empírica e posteriormente "tratados" com base em ferramentas e técnicas. Problemas estão relacionados à percepção dos atores políticos. "Problem definition", diz a autora, "is never simply a 
matter of defining goals and measuring our distance from them. It is rather the strategic representation of situations" (Stone, 2002, 133). A definição de um problema é uma representação porque toda descrição de uma situação prioriza um ponto de vista, entre vários outros possíveis. E é estratégica porque essa descrição é deliberadamente escolhida para promover um determinado curso de ação. A definição de problemas envolve, portanto, disputa entre os atores sobre conceitos, classificações e delimitações sobre as ideias. No discurso político, existem diversas formas de representar estrategicamente um problema e cada uma dessas formas é entendida por Stone $(1989 ; 2002)$ como uma linguagem específica. A linguagem, seja na forma simbólica ou numérica (categorias identificadas e analisadas pela autora), são ferramentas nas mãos dos atores envolvidos numa política que procurarão definir o problema a ser enfrentado pelo governo de forma a privilegiar as ações que preferem desenvolver, ou seja cada ator desenvolverá uma representação de mundo que favoreça suas habilidades, conhecimentos e curso de ação.

\section{Tendências na análise das políticas públicas}

As críticas dos autores sobre a perspectiva racionalista da análise de políticas públicas e as contribuições dos debates travados no contexto da "guinada argumentativa" influenciaram uma série de desenvolvimentos teóricos no campo de políticas públicas. Teorias que incorporam o papel das ideias foram desenvolvidas nos anos 1990 para explicar o processo de produção de políticas, combinando elementos como instituições, atores, ideias, fatores socioeconômicos, políticos, entre outros.

O modelo teórico desenvolvido por John Kingdon (2003) para a análise da formulação de políticas governamentais reserva às ideias papel central. Kingdon (2003) procura analisar como questões chegam à agenda, ou seja, como algumas ideias se tornam importantes num determinado momento, chegando à consideração efetiva dos formuladores de políticas. Ideias exercem 
influência sobre a agenda governamental em dois diferentes momentos do processo de formulação de políticas públicas: na definição de problemas que serão discutidos e na escolha de ações a serem colocadas em prática pelo governo.

Partindo do pressuposto de que os problemas não são passíveis de apreensão por meio de indicadores objetivos, Kingdon (2003), assim como Stone (2002) procura mostrar que problemas são construções sociais, estabelecidas por meio da percepção dos participantes envolvidos no processo de produção de políticas. Para tanto, o autor estabelece uma importante diferenciação entre problemas e questões (conditions). Uma questão (condition) é uma situação social percebida, mas que não desperta necessariamente uma ação governamental. Estas questões se configuram como problemas apenas quando os formuladores de políticas acreditam que devem fazer algo a respeito. Portanto é por meio da argumentação e da coleta de evidências que questões se configuram enquanto problemas, podendo assim chegar à agenda governamental. Esse processo pode se dar por meio de uma seleção de indicadores capazes de sustentar interpretações sobre uma questão específica, definindo-a como uma situação que demanda atuação governamental. Em outros casos, problemas são reconhecidos devido a crises, desastres ou eventos de grande magnitude que ajudam a concentrar a atenção num determinado assunto. A forma como um problema é definido, articulado, concentrando a atenção dos formuladores de política pode determinar o sucesso de uma questão no processo altamente competitivo de agenda-setting, no qual diversas outras questões estão colocadas, aguardando a atenção destes formuladores.

Ideias são essenciais também para explicar como alternativas são selecionadas no processo de formulação de políticas. Alternativas são as soluções, ou ideias a respeito do que fazer sobre uma determinada questão, geradas em comunidades de especialistas (policy communities), compostas por pesquisadores, assessores parlamentares, acadêmicos, funcionários públicos, analistas pertencentes a grupos de interesses, entre outros, - que compartilham uma preocupação em relação a uma política. Nessas 
comunidades, as ideias são testadas: algumas delas sobrevivem aos debates sem grandes alterações, outras se confrontam e se combinam em novas propostas; outras ainda são descartadas. Neste processo competitivo de seleção, as ideias que se mostram viáveis do ponto de vista técnico e as que têm custos toleráveis geralmente sobrevivem, assim como aquelas que representam valores compartilhados, contam com a aceitação do público em geral e com a receptividade dos formuladores de políticas. Como resultado final, partindo de um grande número de ideias possíveis, um pequeno conjunto de alternativas emerge para a efetiva consideração dos participantes do processo decisório. Quando uma proposta é percebida como viável, esta é rapidamente difundida, ampliando a consciência dos atores sobre uma determinada ideia. Isso não significa que todos os especialistas de uma comunidade compartilhem das mesmas crenças: algumas comunidades são extremamente fragmentadas, abrigando pontos de vista bastante diversificados. A difusão das ideias tão pouco se dá de forma automática, uma vez que comunidades bem estruturadas apresentam tendência a resistirem às novas ideias. A difusão é descrita pelo autor como um processo no qual indivíduos que defendem uma ideia procuram levá-la a diferentes fóruns, na tentativa de sensibilizar não apenas as comunidades de políticas, mas também o público em geral, habituando a audiência às propostas e construindo progressivamente suporte para sua aceitação. Desta forma, as ideias são difundidas, basicamente, por meio da persuasão. Desta forma, Kingdon (2003: 125) assegura às ideias um papel importante em seu modelo, argumentando que estas são frequentemente mais importantes na escolha de uma alternativa do que a influência de fatores mais comumente analisados como, por exemplo, a atuação dos grupos de pressão.

Quando bem construídas, argumentadas e defendidas, as ideias a respeito de problemas e de alternativas podem chegar à agenda em contextos políticos favoráveis. Sob algumas circunstâncias, a dinâmica política pode engendrar aquilo que o autor denomina como "clima" ou "humor" nacional favorável ou, ao contrário, limitar o acesso de algumas questões à agenda. 
Momentos de mudanças dentro do governo, com alterações em indivíduos em posições estratégicas dentro da estrutura governamental, por exemplo, podem potencializar a emergência de algumas ideias, da mesma forma que podem também limitar outras.

Outro modelo teórico reserva às ideias papel central na explicação sobre a produção de políticas públicas: a perspectiva do equilíbrio pontuado (punctuated equilibrium), desenvolvido por Baumgartner e Jones (1993). Os autores desenvolveram esse modelo para explicar períodos de estabilidade, característicos do sistema político e administrativo, pontuados por momentos de rápida mudança. Para explicar as mudanças em políticas púbicas, os autores elaboram o conceito de "imagens de políticas" (policy image), que corresponde à forma como uma política é compreendida e discutida (Baumgartner e Jones, 1993, 25). As imagens são as ideias que sustentam os arranjos institucionais, permitindo que o entendimento acerca da política seja comunicado de forma simples e direta entre os membros de uma comunidade, contribuindo para a disseminação das questões. Quando uma imagem é largamente compartilhada e aceita, estrutura-se um "monopólio de políticas". Os monopólios são arranjos constituídos com base em duas características: uma estrutura institucional definida, pela qual o acesso de atores ao processo decisório é permitido (ou restringido); e uma ideia fortemente associada a essa estrutura institucional. As imagens, portanto, dão sustentação a um monopólio de políticas. Por outro lado, quando há divergências com relação ao entendimento de uma política, defensores de uma ideia focalizam determinadas imagens enquanto seus oponentes podem se concentrar num conjunto diferente de imagens, o que pode levar à desestabilização ou mesmo ao colapso do monopólio.

As imagens são desenvolvidas com base em dois componentes: informações empíricas e apelos emotivos (tone). O tone é considerado pelos autores como fator crítico no desenvolvimento das questões, uma vez que mudanças rápidas nesses "apelos emotivos" da imagem podem influenciar a mobilização em torno de uma ideia. Os autores exemplificam este 
tipo de situação na questão nuclear norte-americana: enquanto a imagem predominante estava associada ao progresso econômico e científico, um monopólio existia em torno desta questão. No entanto, a partir do momento em que a imagem muda, focalizando as ameaças de segurança e degradação ambiental, o monopólio em torno desta questão se esgotou. Novas imagens podem atrair novos participantes (ou afastá-los), bem como criar oportunidades para promover questões (ou desencorajar outras). A disputa em torno do entendimento de uma política - ou da imagem - é considerada pelos autores como sendo elemento crucial na luta política. A mudança em políticas públicas se processa, segundo os autores, devido a alterações nas ideias subjacentes às políticas. Mudanças na percepção das questões, sobretudo quando estas passam a ser compreendidas como problemas, a ocorrência de eventos que focalizem atenção da opinião púbica e do governo, entre outros fatores, criam oportunidades para que diferentes ideias passem a chamar a atenção dos formuladores de políticas, rompendo os "monopólios", ou seja, arranjos baseados em regras e ideias que mantém o sistema em equilíbrio. Novas ideias - e novos atores - podem estabelecer novas imagens e promover reorganizações. Essas novas ideias e instituições tendem a permanecer no tempo, criando um novo estado de equilíbrio que, após algum tempo, pode conformar um novo monopólio de política, voltando à estabilidade. Assim, para os autores, a chave para a compreensão dos períodos de estabilidade e mudança em uma política pública reside no plano das ideias: na forma como uma questão é definida, considerando que esta definição se desenvolve dentro de um contexto institucional que pode favorecer determinadas visões políticas em detrimento de outras.

Um terceiro modelo teórico também reserva especial destaque às ideias na explicação do processo de produção de políticas públicas: o modelo de "coalizões de defesa", de Sabatier e Jenkins-Smith (1993). Uma das premissas desse modelo consiste no entendimento das políticas públicas como um sistema de crenças. Segundo os autores, políticas e programas de governo incorporam teorias implícitas sobre como atingir seus objetivos, envolvendo 
ideias, valores, percepções sobre causas e efeitos, percepção sobre a eficácia dos instrumentos políticos, entre outras.

Segundo o modelo, os atores envolvidos com uma política podem formar coalizões - compostas por indivíduos oriundos tanto do interior da estrutura governamental quanto do setor privado. O elemento que mantém esses atores unidos é um conjunto de crenças em comum, além de seu empenho em ações coordenadas em prol de uma política. $\mathrm{O}$ conjunto de crenças dessas coalizões apresenta três componentes básicos. Num plano mais geral e abstrato, estão as deep core beliefs, que incluem valores ontológicos e normativos, sendo este tipo de crença altamente resistente à mudança. Abaixo destas estão as policy core beliefs, um pouco menos rígidas que as primeiras, e que representam as percepções comuns sobre os valores compartilhados em torno da política de interesse. Mudanças nas policy core beliefs representam as maiores mudanças possíveis numa política. Finalmente, um terceiro componente são crenças mais específicas, relacionadas à visão do problema e à importância dos fatores causais desses problemas, bem como o desenho das instituições, avaliação do desempenho dos atores e alocações orçamentárias. Alterações neste grupo de crenças secundárias representam mudanças menores (minor policy change). As coalizões, desta forma, se mantêm unidas em torno de crenças; crenças que orientam sua ação no processo de produção de políticas públicas.

Para os autores, podemos encontrar diversas coalizões associadas a uma política (geralmente entre duas e quatro coalizões) que compartilham crenças mas que, em determinados momentos, adotam estratégias diferentes para realizar seus objetivos de mudança. Esta competição entre as coalizões gera disputas e, como resultado dessa dinâmica, as políticas governamentais são produzidas. Este processo altera também a própria ação das coalizões, num processo que, por meio da percepção de adequação das decisões governamentais, dos impactos produzidos, e de novas informações, gera um aprendizado constante (Sabatier e Jenkins-Smith, 1999: 123). Aplicada em diversos estudos sobre política ambiental, educação, defesa, energia, regulação de 
telecomunicações, infraestrutura, entre outras políticas, o modelo fornece recursos metodológicos para mapear os sistemas de crenças das coalizões.

Os três modelos teóricos sinteticamente apresentados nessa seção procuram mostrar que a produção de políticas públicas é caracterizada pela interação de valores, normas, crenças e diferentes formas de conhecimento, trazendo ao centro da análise a questão das ideias. Ao invés de alinharem-se à formulações tradicionais da área de políticas públicas que concentram sua atenção em questões como poder, influência, pressão e estratégia, os modelos apontam o desenvolvimento e a difusão das ideias como elemento central do processo político. Embora compartilhem das mesmas premissas, os modelos aqui abordados recorrem a recursos metodológicos distintos. De qualquer forma, ao oferecer mecanismos para explorar a influência das crenças, argumentos e representações no processo político, a aplicação desses modelos tem permitido ampliar o entendimento sobre o papel das ideias na produção de políticas públicas.

\section{Considerações finais}

Este estudo procurou apresentar uma visão panorâmica sobre o desenvolvimento do campo de políticas públicas, partindo das contribuições seminais de Lasswell, na década de 1950, passando pela "guinada analítica" dos anos 1960 a 1980, até chegar na "guinada argumentativa" que teve início no começo dos anos 1990. Neste percurso, procuramos mostrar que os estudos mais recentes em políticas públicas têm reservado às ideias papel central em seus mecanismos explicativos. No entanto, não basta constatar a crescente importância das ideias na literatura especializada, cabe discutir como as ideias influenciam na produção de políticas. Parte da resposta vem sendo desenvolvida nos modelos teóricos desenvolvidos no campo, analisados na seção anterior. As ideias estão no centro das explicações sobre mudanças na agenda governamental, exercendo influência sobre dois importantes 
processos no contexto da formulação de políticas: a definição dos problemas e a seleção de alternativas (Kingdon, 2003). As ideias também auxiliam na compreensão de períodos de mudança e de estabilidade em políticas, por meio de "imagens" que, juntamente com instituições, promovem tanto situações de equilíbrio quanto de ruptura em políticas públicas (Baumgartner e Jones, 1993). Ideias também mantêm indivíduos unidos em coalizões que, em disputa, produzirão mudanças em políticas públicas (Sabatier e JenkinsSmith, 1999). Cada modelo, com suas opções metodológicas, oferece alternativas de operacionalização de análises de políticas públicas centradas nas ideias.

Uma das críticas mais comuns às abordagens centradas nas ideias diz respeito à definição precisa do que sejam, afinal, as ideias. As perspectivas de Majone (1989), Stone (2002) assumem que ideias são argumentos. No debate público, envolvendo amplas audiências, ideias se formam e políticas são moldadas no processo dialógico de argumentação e persuasão. Essa é uma perspectiva bastante diferente de assumir a argumentação como racionalização, no qual a linguagem seria meramente um veículo pelo qual ideias vinculadas a interesses se manifestam. Apesar disso, o sentido das ideias, enquanto conceito, permanece impreciso se considerarmos os modelos teóricos mais recentes, que tendem a assumir uma visão das ideias bastante associada à crença dos atores a respeito de características associadas a uma política, suas visões de mundo e opiniões. Nesse sentido, uma questão fundamental parece residir no aprofundamento desse conceito. Esse esforço pode contribuir para novos entendimentos sobre o processo de produção de políticas e sobre o próprio campo de políticas públicas.

\section{Bibliografia}

BAUMGARTNER, Frank R; JONES, Bryan D. Agendas and Instability in American Politics. Chicago, University of Chicago Press, 1993. 
(1999). "Punctuated Equilibrium Theory: Explaining stability and change in american policymaking". In Sabatier, Paul A. (ed.). Theories of The Policy Process. Oxford, Westview Press. (pp. 97-116).

CAPELLA, Ana C. N. A "Linguagem da Administração Pública: um estudo sobre a abordagem pós-moderna". Cadernos Gestão Pública e Cidadania. São Paulo, v. 16 nº 59, jul-dez 2011.

DeLEON, Peter. "The stages approach to the policy process: what has it done? Where is it going?" In Sabatier, Paul (ed.). Theories of the Policy Process. Oxford, Westview Press, 1999.

. "The Historical Roots of the Field". In Moran, M. et. all. The Oxford Handbook of Public Policy. New York, Oxford University Press, 2008.

DUNN, William N. Public Policy Analysis. Englewood Cliffs, New Jersey: Prentice-Hall, 1994.

FARAH, Marta F. S. "A contribuição da administração pública para a constituição do campo de estudos de políticas públicas". In Marques, E. e Faria, C.A.P. A política pública como campo multidisciplinar. São Paulo/Rio de Janeiro: Unesp/Fiocruz, 2013.

FARIA, Carlos A. P. "Ideias, Conhecimento e Políticas Públicas: Um inventário sucinto das principais vertentes analíticas recentes". Revista Brasileira de Ciências Sociais, vol. 18, no. 51, fevereiro 2003. (pp. 21-29).

FISCHER, Frank. Handbook of Public Policy: Theory, Politics and Methods. Boca Raton-FL, 2007.

FISHER, Frank e FORESTER, John. The Argumentative Turn in Policy Analysis and Planning. London, Duke University Press, 1993.

HALL, Peter. "Policy paradigms, social learning, and the state: the case of economic policymaking in Britain". Comparative Politics, vol. 25, no 3, 1993 (275-296). 
HILL, Michael. The Public Policy Process. Harlow, England: Pearson/ Longman, 2009.

JOHN, Peter. "Is There Life After Policy Streams, Advocacy Coalitions, and Punctuations: Using Evolutionary Theory to Explain Policy Change?". Policy Studies Journal, 31 (4), 481498, 2003.

Analyzing Public Policy. 2nd. Ed. London: Routledge, 2012.

KINGDON, John W. Agendas, Alternatives, and Public Policies. 2nd. Ed. New York, Longman, [1984]. 2003.

LASSWELL, Harold. “The Policy Orientation”. In Lerner, D e Lasswell, H. The Policy Sciences: recent developments in scope and method. Stanford: Stanford University Press, 1951.

MAJONE, Giandomenico. Evidence, Argument \& Persuasion in the Policy Process. New Haven/London, Yale University Press, 1989.

. "Public policy and administration: ideas, interests and institutions". In Goodin, Robert E. and Klingemann, Hans-Dieter, eds., A New Handbook of Political Science. Oxford: Oxford University Press (pp., 610-627), 1998.

MORAN, M. et. all. The Oxford Handbook of Public Policy. New York, Oxford University Press, 2008.

SABATIER, Paul A. e JENKINS-SMITH, Hank C. Policy Change and Learning: An Advocacy Coalition Approach. Oxford, Westview Press, 1993.

SABATIER, Paul A. e WEIBLE, Christopher M. "The Advocacy Coalition: Innovations and Clarifications." In Sabatier, P. (ed.).Theories of the Policy Process. 2 ${ }^{\underline{a}}$ ed. Boulder, CO: Westview Press, 2007.

SMITH, Kevin B. e LARIMER, Christopher W. The Public Policy Theory Primer. Boulder-CO, Westview Press, 2009. 
Análise de políticas públicas...

STONE, Deborah A. "Causal Stories and the Formation of Policy Agendas". Political Science Quarterly. Vol. 104. No. 2. (pp. 281300), 1989.

Policy Paradox: The Art of Political Decision Making. Nova York, Norton \& Company, [1988]. 2002. 